Journal of Universal Mathematics

Vol.2 No.2 PP.127-136 (2019)

ISSN-2618-5660

\title{
QUANTUM CODES FROM CODES OVER THE RING
}

\author{
$\mathbb{F}_{2^{m}}+\alpha \mathbb{F}_{2^{m}}+\beta \mathbb{F}_{2^{m}}+\gamma \mathbb{F}_{2^{m}}$ \\ MURAT GÜZELTEPE AND MUSTAFA ERÖZ
}

\begin{abstract}
Let $i, j, k$ be elements of real quaternions $\mathbb{H}$. Let $\alpha, \beta, \gamma$ be the elements corresponding to $1+i, 1+j, 1+k$, respectively. In this study, quantum codes from classical codes over $\mathbb{F}_{2^{m}}+\alpha \mathbb{F}_{2^{m}}+\beta \mathbb{F}_{2^{m}}+\gamma \mathbb{F}_{2^{m}}$ are obtained.
\end{abstract}

\section{INTRODUCTION}

The relationship between quantum codes and classical codes has been discussed by the authors since the first quantum error correcting codes obtained. It was shown in [1] that quantum codes can be obtained from classical self-orthogonal codes. Thms. 1-3 in [1] have been used by many researchers to establish a bridge between quantum codes and classical codes. Many researchers have utilized different finite fields and finite rings to construct quantum codes. For example, regarding Gray image of linear or cyclic codes over $\mathbb{F}_{4}+u \mathbb{F}_{4}$, some quantum codes were presented in [2]. A different method to obtain quantum error-correcting codes from cyclic codes over $\mathbb{F}_{2}+v \mathbb{F}_{2}$ was given in [3].

On the other hand, linear and cyclic codes over some special rings were defined by some researchers. In [4], codes over the ring $\mathbb{Z}_{2 m}+\alpha \mathbb{Z}_{2 m}+\beta \mathbb{Z}_{2 m}+\gamma \mathbb{Z}_{2 m}$, in [5], Cyclic codes over $\mathbb{F}_{2}+u \mathbb{F}_{2}+v \mathbb{F}_{2}+u v \mathbb{F}_{2}$ were defined.

In this paper, we construct quantum codes via codes over the $\operatorname{ring} \mathbb{F}_{2^{m}}+\alpha \mathbb{F}_{2^{m}}+$ $\beta \mathbb{F}_{2^{m}}+\gamma \mathbb{F}_{2^{m}}$. Some of these quantum codes are better than previous ones. We prefer the ring $\mathbb{F}_{2^{m}}+\alpha \mathbb{F}_{2^{m}}+\beta \mathbb{F}_{2^{m}}+\gamma \mathbb{F}_{2^{m}}$ to the ring $\mathbb{F}_{2^{m}}+u \mathbb{F}_{2^{m}}+v \mathbb{F}_{2^{m}}+u v \mathbb{F}_{2^{m}}$, where $u^{2}=v^{2}=0, u v=v u$ since, using a norm function, one can easily determine the minimum distance of a code.

In what follows, we consider the following:

Shortly, we take the finite field $\mathbb{F}_{2^{m}}$ as a field extension of $\mathbb{F}_{2}$ such that

$$
\mathbb{F}_{2^{m}}=\mathbb{Z}_{2}[x] /(p(x))
$$

where $p(x)$ is an irreducible monic polynomial in $\mathbb{Z}_{2}[x]$ of degree $m$. The Gray map $\phi$ from $\mathbb{F}_{2^{m}}$ to $\mathbb{F}_{2}^{m}$ is defined as $\phi\left(a_{0}+a_{1} x+\cdots+a_{m-1} x^{m-1}\right)=\left(\begin{array}{cccc}a_{0}, & a_{1}, & \ldots, & a_{m-1}\end{array}\right)$.

2000 Mathematics Subject Classification. 94B05, 94B15, 94B35, 94B60.

Key words and phrases. Block codes, Quantum codes.

This study is supported by TÜBİTAK under the project number 116F318. 
For example, let $p(x)=x^{3}+x+1$ then

$$
\mathbb{F}_{2^{3}}=\left\{a_{0}+a_{1} x+a_{2} x^{2}: a_{0}, a_{1}, a_{2} \in \mathbb{F}_{2}\right\}
$$

and hence we get $\phi(0)=\left(\begin{array}{lll}0, & 0, & 0\end{array}\right), \phi(1)=\left(\begin{array}{lll}1, & 0, & 0\end{array}\right), \phi(1+x)=\left(\begin{array}{lll}1, & 1, & 0\end{array}\right)$, etc. This Gray map can naturally be extended to $\mathbb{F}_{2^{m}}^{n}$ by applying it coordinatewise.

The Hamming weight of a vector $u \in \mathbb{F}_{2}^{m}$ is defined as the number of nonzero components and the Hamming weight of a vector $u \in \mathbb{F}_{2^{m}}$ is naturally defined as the number of nonzero components of $\phi(u)$ and denoted by $w t(\phi(u))$.

Definition 1.1. [6] The Hamilton Quaternion Algebra over the set of the real numbers $(\mathbb{R})$, denoted by $H(\mathbb{R})$, is the associative unital algebra given by the following representation:

i) $H(\mathbb{R})$ is the free $\mathbb{R}$ module over the symbols $1, i, j, k$, that is, $H(\mathbb{R})=\left\{a_{0}+a_{1} i+\right.$ $\left.a_{2} j+a_{3} k: a_{0}, a_{1}, a_{2}, a_{3} \in \mathbb{R}\right\}$

ii) 1 is the multiplicative unit;

iii) $i^{2}=j^{2}=k^{2}=-1$;

iv) $i j=-j i=k, k i=-i k=j, j k=-k j=i$.

From here onwards, $R_{2 m}$ denotes the ring

$\mathbb{Z}_{2 m}+\alpha \mathbb{Z}_{2 m}+\beta \mathbb{Z}_{2 m}+\gamma \mathbb{Z}_{2 m}$.

\section{THE RING $R_{2^{m}}$}

$R_{2^{m}}=\mathbb{F}_{2^{m}}+\alpha \mathbb{F}_{2^{m}}+\beta \mathbb{F}_{2^{m}}+\gamma \mathbb{F}_{2^{m}}$ is a commutative ring. The size of this ring is $2^{4 m}$, that is, $\left|R_{2}^{m}\right|=2^{4 m}$. The ring $R_{2^{m}}$ is a local ring. But it is not a principal ideal ring or finite chain. The ring $R_{2}$ has only one maximal ideal which is not principal. The maximal ideal is $\langle\alpha\rangle \oplus\langle\beta\rangle=\{0,1+i, 1+j, 1+k, i+j, i+k, j+k, 1+i+j+k\}$. The ideals of the ring $R_{2}$ can be introduced by

$$
\begin{gathered}
\langle 0\rangle=\{0\} \subset\langle 1+i+j+k\rangle=\{0,1+i+j+k\} \subset\langle\alpha\rangle \\
=\{0,1+i, j+k, 1+i+j+k\},\langle\beta\rangle,\langle\gamma\rangle \subset\langle\alpha\rangle \oplus\langle\beta\rangle \\
=\langle\alpha\rangle \oplus\langle\gamma\rangle=\langle\beta\rangle \oplus\langle\gamma\rangle \subset\langle 1\rangle=R_{2} .
\end{gathered}
$$

Note that $R_{2} /\langle\alpha\rangle \oplus\langle\beta\rangle$ is isomorphic to $\mathbb{F}_{2}$ and $\langle\alpha+\beta\rangle=\langle\gamma\rangle$.

The followings were defined in [4]:

- The conjugate of an element $q=a+b \alpha+c \beta+d \gamma$ in $R_{2^{m}}$ is $\bar{q}=a+b \bar{\alpha}+$ $c \bar{\beta}+d \bar{\gamma}$.

- $\alpha \bar{\alpha}=\beta \bar{\beta}=\gamma \bar{\gamma}=2$.

- The norm of $q=a+b \alpha+c \beta+d \gamma$ is

$N(q)=q \bar{q}=a^{2}+2 b^{2}+2 c^{2}+2 d^{2}+2 a b+2 a c+2 a d+2 b c+2 b d+2 c d$.

The norm of a vector $u=u_{t} \in R_{2^{m}}^{n}$ was given by $\sum N\left(u_{t}\right)$.

It should be noted that if $u \in R_{2^{m}}-(\langle\alpha\rangle \oplus\langle\beta\rangle), v \in\langle\alpha\rangle \oplus\langle\beta\rangle$ then $N(u) \in$ $\mathbb{F}_{2^{m}}-\{0\}, N(v)=0$.

\section{A Gray map}

Let $\psi_{2^{m}}: \mathbb{F}_{2^{m}}+\alpha \mathbb{F}_{2^{m}}+\beta \mathbb{F}_{2^{m}}+\gamma \mathbb{F}_{2^{m}} \rightarrow \mathbb{F}_{2^{m}}^{4}$ by

$$
\psi_{2^{m}}(a+b \alpha+c \beta+d \gamma)=(b, \quad c, \quad d, \quad a+b+c+d .)
$$


The map $\psi_{2^{m}}$ can naturally be extended to $R_{2^{m}}^{n}$ by applying it coordinatewise. The Euclidean weight of a vector $u=\left(u_{t}\right) \in \mathbb{F}_{2^{m}}^{n}$ is given by $w(u)=\sum\left(u_{t}\right)^{2}$. For example, for $m=2, p(x)=x^{2}+x+1$, then

$$
\begin{aligned}
& \mathbb{F}_{4}+\alpha \mathbb{F}_{4}+\beta \mathbb{F}_{4}+\gamma \mathbb{F}_{4}=\left\{a+b \alpha+c \beta+d \gamma: a, b, c, d \in F_{4}\right\},
\end{aligned}
$$

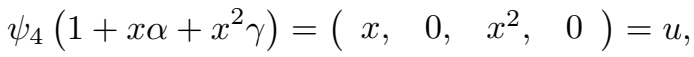

$$
\begin{aligned}
& \sum_{t=0}^{3}\left(u_{t}\right)^{2}=x^{2}+0^{2}+\left(x^{2}\right)^{2}+0^{2}=x^{2}+x^{4}=x^{2}+x=1 \\
& \psi(1)=(1,0), \quad w t((1,0))=1 .
\end{aligned}
$$

On the other hand the norm of $1+x \alpha+x^{2} \gamma=1+x(1+i)+x^{2}(1+k)=1+x+$ $x^{2}+x i+x^{2} k=x i+x^{2} k$ is calculated as

$$
N\left(x i+x^{2} k\right)=x^{2}+x^{4}=1 .
$$

Note $1+x+x^{2}=0$ and $x^{3}=1$ in $\mathbb{F}_{4}$.

Theorem 3.1. If $u$ is a vector in $R_{2^{m}}^{n}$ then $N(u)=w\left(\psi_{2^{m}}(u)\right)$ implies that $w t(N(u))=w t\left(w\left(\psi_{2^{m}}(u)\right)\right)$.

Proof. Let $u=q=a_{0}+a_{1} \alpha+a_{2} \beta+a_{3} \gamma \in R_{2^{m}}$, where $a_{0}, a_{1}, a_{2}, a_{3} \in \mathbb{F}_{2^{m}}$. Then the norm of $N(u=q)=q \bar{q}=a_{0}^{2}+2 a_{1}^{2}+2 a_{2}^{2}+2 a_{3}^{2}+2 a b+2 a c+2 a d+2 b c+2 b d+2 b c$. On the other hand, $\psi_{2^{m}}\left(a_{0}+a_{1} \alpha+a_{2} \beta+a_{3} \gamma\right)=\left(a_{1}, a_{2}, a_{3}, a_{0}+a_{1}+a_{2}+a_{3}\right)=$ $\left(u_{0}, u_{1}, u_{2}, u_{3}\right)=u$. Hence we get

$$
\sum_{t=0}^{3}\left(u_{t}\right)^{2}=a_{1}^{2}+a_{2}^{2}+a_{3}^{2}+\left(a_{0}+a_{1}+a_{2}+a_{3}\right)^{2}=N(u) .
$$

If $u$ is taken $\left(q_{1}, q_{2}, \ldots, q_{n}\right) \in R_{2^{m}}^{n}$, it is obtained that $N(u)=w\left(\psi_{2^{m}}(u)\right)$ by applying the same method to each component.

The proof of the next theorem is clear from the Gray map and Thm. 1.

Theorem 3.2. The function $\psi_{2^{m}}$ is linear and bijective.

\section{Linear CODES OVER $R_{2^{m}}$}

We start to determine the ideals of the ring $R_{2^{m}}$. As for the ideal structure we can find the ideals of $R_{2^{m}}$ to be listed as

$$
\begin{gathered}
\langle 0\rangle=\{0\} \\
\langle\alpha+\beta+\gamma\rangle=(\alpha+\beta+\gamma) R_{2^{m}}, \quad|\langle\alpha+\beta+\gamma\rangle|=2^{m} \\
\langle\alpha\rangle=(\alpha) R_{2^{m}}, \quad|\langle\alpha\rangle|=2^{2 m} \\
\langle\beta\rangle=(\beta) R_{2^{m}}, \quad|\langle\beta\rangle|=2^{2 m} \\
\langle\alpha\rangle+\langle\beta\rangle=(\alpha) R_{2^{m}}+(\beta) R_{2^{m}},|\langle\alpha\rangle+\langle\beta\rangle|=2^{3 m} .
\end{gathered}
$$

Note that

$$
R_{2^{m}} /(\langle\alpha\rangle+\langle\beta\rangle)
$$

is isomorphic to the field $\mathbb{F}_{2^{m}}$ with the characteristic 2 and

$$
\langle\alpha\rangle \neq\langle\beta\rangle,\langle\alpha+\beta\rangle=\langle\gamma\rangle \text {. }
$$


The ideal $\langle\alpha\rangle+\langle\beta\rangle$ is a maximal ideal of $R_{2^{m}}$ and $R_{2^{m}}$ has only one maximal ideal. So, $R_{2^{m}}$ is a local ring. It is well known from algebra that if a ring $R$ is a local ring with maximal ideal $M$ then an element in $R-M$ is a unit in $R$. Hence, we can determine the units of $R_{2^{m}}$ as

$$
R_{2^{m}}^{*}=R_{2^{m}}-(\langle\alpha\rangle+\langle\beta\rangle),\left|R_{2^{m}}^{*}\right|=\left(2^{3 m}\right)\left(2^{m}-1\right) .
$$

Definition 4.1. A linear code $C$ of length $n$ over the ring $R_{2^{m}}$ is an $R_{2^{m}}$-submodule of $R_{2^{m}}^{n}$.

To classify the generators for linear codes over $R_{2^{m}}$ we must determine linear independence condition of them to establish possible type for linear codes over $R_{2}$. There are six type generators for linear codes over $R_{2^{m}}$ such that we determine them as $[a],[b],[c],[d],[e],[f]$. Here,

$$
\begin{gathered}
{[a] \in R_{2^{m}}^{n} \backslash(\langle\alpha\rangle+\langle\beta\rangle)^{n}} \\
{[b] \in(\langle\alpha\rangle+\langle\beta\rangle)^{n},[b] \notin(\langle\alpha\rangle)^{n},(\langle\beta\rangle)^{n},(\langle\gamma\rangle)^{n}} \\
{[c] \in(\langle\alpha\rangle)^{n} \backslash(\langle\alpha+\beta+\gamma\rangle)^{n}} \\
{[d] \in(\langle\beta\rangle)^{n} \backslash(\langle\alpha+\beta+\gamma\rangle)^{n}} \\
{[e] \in(\langle\gamma\rangle)^{n} \backslash(\langle\alpha+\beta+\gamma\rangle)^{n}} \\
{[f] \in(\langle\alpha+\beta+\gamma\rangle)^{n} .}
\end{gathered}
$$

Theorem 4.2. If $C$ is a linear code over $R_{2^{m}}$ then the size of the code $C$ is $(2)^{4 m k_{1}}(2)^{3 m k_{2}}(2)^{2 m k_{3}}(2)^{2 m k_{4}}(2)^{2 m k_{5}}(2)^{m k_{6}}$.

The proof is straightforward from above generators.

Theorem 4.3. If $C$ is a linear code over $R_{2^{m}}$ of length $n$, size $2^{k}$ and minimum Euclidean distance $d$, then $\psi(C)$ is a $[4 n, k, d]$-linear code over $\mathbb{F}_{2^{m}}$ and $\phi(\psi(C))$ is a binary $[4 m n, k, d]$-linear code over $\mathbb{F}_{2}$.

Recall that the maps $\psi_{2^{m}}$ and $\phi$ are linear and bijective. So, the proof is straightforward.

Before we give a relationship between self-dual codes over $R_{2^{m}}$ and self-dual codes over $\mathbb{F}_{2^{m}}$ and $\mathbb{F}_{2}$, we must give the inner product in $R_{2^{m}}^{n}$.

We define a similar inner product in [4] such $\langle u, v\rangle=\phi\left(\sum_{t=1}^{n} u_{t} \overline{v_{t}}\right)$, where $u=\left(u_{1}, u_{2}, \ldots, u_{n}\right), v=\left(v_{1}, v_{2}, \ldots, v_{n}\right) \in R_{2^{m}}^{n}$. Recall that the ring $R_{2^{m}}$ is commutative. So, $\langle u, v\rangle=\langle v, u\rangle$.

Theorem 4.4. If $C$ is a self-dual code of length $n$ over $R_{2^{m}}$, then $\psi_{2^{m}}(C)$ is a self-dual code of length $4 n$ over $\mathbb{F}_{2^{m}}$ and $\phi\left(\psi_{2^{m}}(C)\right)$ is a self-dual code of length 4 mn over $\mathbb{F}_{2}$.

Proof. Let $C$ be a self-dual code over $R_{2^{m}}$ and let $u, v$ be vectors in $C$. Let $u=$ $\left(u_{1}, u_{2}, \ldots, u_{n}\right), v=\left(v_{1}, v_{2}, \ldots, v_{n}\right) \in C$, shortly $u_{t}=a_{t}+b_{t} \alpha+c_{t} \beta+d_{t} \gamma, v_{t}=$ $a_{t}^{\prime}+b_{t}^{\prime} \alpha+c_{t}^{\prime} \beta+_{t}^{\prime} \gamma$. It is obvious that

$$
\begin{aligned}
& \langle u, v\rangle=0(\bmod p(x))=\sum_{t}\left(a_{t}+b_{t} \alpha+c_{t} \beta+d_{t} \gamma\right)\left(a_{t}^{\prime}+b_{t}^{\prime} \alpha+c_{t}^{\prime} \beta+d_{t}^{\prime} \gamma\right) \\
& =\sum_{t}\left(a_{t}+b_{t}+c_{t}+d_{t}+b_{t} i+c_{t} j+d_{t} k\right)\left(a_{t}^{\prime}+b_{t}^{\prime}+c_{t}^{\prime}+d_{t}^{\prime}+b_{t}^{\prime} i+c_{t}^{\prime} j+d_{t}^{\prime} k\right) \\
& \sum_{t}\left(\begin{array}{c}
\left(a_{t}+b_{t}+c_{t}+d_{t}\right)\left(a_{t}^{\prime}+b_{t}^{\prime}+c_{t}^{\prime}+d_{t}^{\prime}\right)+\left(a_{t}+b_{t}+c_{t}+d_{t}\right)\left(b_{t}^{\prime} i+c_{t}^{\prime} j+d_{t}^{\prime} k\right) \\
+\left(b_{t} i+c_{t} j+d_{t} k\right)\left(a_{t}^{\prime}+b_{t}^{\prime}+c_{t}^{\prime}+d_{t}^{\prime}\right)+\left(b_{t} i+c_{t} j+d_{t} k\right)\left(b_{t}^{\prime} i+c_{t}^{\prime} j+d_{t}^{\prime} k\right)
\end{array}\right)
\end{aligned}
$$


On the other hand, we get

$$
\begin{aligned}
& \left\langle\psi_{2^{m}}(u), \psi_{2^{m}}(v)\right\rangle=\sum_{t}\left(\psi_{2^{m}}\left(a_{t}+b_{t} \alpha+c_{t} \beta+d_{t} \gamma\right)\right)\left(\psi_{2^{m}}\left(a_{t}^{\prime}+b_{t}^{\prime} \alpha+c_{t}^{\prime} \beta+d_{t}^{\prime} \gamma\right)\right) \\
& =\sum_{t}\left(\begin{array}{llll}
b_{t}, & c_{t}, & d_{t}, & a_{t}+b_{t}+c_{t}+d_{t}
\end{array}\right)\left(\begin{array}{cccc}
b_{t}^{\prime}, & c_{t}^{\prime}, & d_{t}^{\prime}, & a_{t}^{\prime}+b_{t}^{\prime}+c_{t}^{\prime}+d_{t}^{\prime}
\end{array}\right) \\
& =\sum_{t} b_{t} b_{t}^{\prime}+c_{t} c_{t}^{\prime}+c_{t} c_{t}^{\prime}+\left(a_{t}+b_{t}+c_{t}+d_{t}\right)\left(a_{t}^{\prime}+b_{t}^{\prime}+c_{t}^{\prime}+d_{t}^{\prime}\right) \\
& \equiv 0(\bmod p(x))
\end{aligned}
$$

Thus we get that $\psi_{2^{m}}(C)$ is a self-orthogonal code over $\mathbb{F}_{2^{m}}$ and $\left|\psi_{2^{m}}(C)\right|=$ $|C|=2^{2 m n}$ since $C$ is a self-dual code. So, $\psi_{2^{m}}(C)$ is a self-dual code of length $4 n$ over $\mathbb{F}_{2^{m}}$. Using the same way, it can be seen that $\phi\left(\psi_{2^{m}}(C)\right)$ is self-dual.

Theorem 4.5. Let $C_{1}, \ldots, C_{6}$ are linear codes over $\mathbb{F}_{2^{m}}$ such that $C_{i} \cap C_{j}=\{0\}$, for $i \neq j$. Let $C$ be a linear code over $R_{2^{m}}$ and let $M$ be the maximal ideal of $R_{2^{m}}$. Then $C$ is expressed as

$C=\left(R_{2^{m}} \backslash M\right) C_{1} \oplus(\langle\alpha\rangle+\langle\beta\rangle) C_{2} \oplus(\langle\alpha\rangle) C_{3} \oplus(\langle\beta\rangle) C_{4} \oplus(\langle\gamma\rangle) C_{5} \oplus(\langle\alpha+\beta+\gamma\rangle) C_{6}$

with the size $|C|=2^{4 m k_{1}+3 m k_{2}+2 m k_{3}+2 m k_{4}+2 m k_{5}+m k_{6}}$ if and only if $C_{1}, \ldots, C_{6}$ are linear codes over $\mathbb{F}_{2^{m}}$, where $k_{1}, \ldots, k_{6}$ denote the dimensions of $C_{1}, \ldots, C_{6}$, respectively. Moreover, if $C$ is a self-orthogonal code then $\psi_{2^{m}}(C)$ is a self-orthogonal code. 5.

The proof is straightforward from the definition of the ideals of $R_{2^{m}}$ and Thm.

The proof of next theorem is clear from Thms. 5,6.

Theorem 4.6. Let

$C=\left(R_{2^{m}} \backslash M\right) C_{1} \oplus(\langle\alpha\rangle+\langle\beta\rangle) C_{2} \oplus(\langle\alpha\rangle) C_{3} \oplus(\langle\beta\rangle) C_{4} \oplus(\langle\gamma\rangle) C_{5} \oplus(\langle\alpha+\beta+\gamma\rangle) C_{6}$

be a linear code over $R_{2^{m}}$ under the conditions above theorem and let

$$
C_{i} \subseteq C_{i-j}^{\perp}, i=6,5,4,3,2, j=1,2,3,4,5 .
$$

If $C_{1}, \ldots, C_{6}$ are self-orthogonal codes then $C$ and $\psi_{2^{m}}(C)$ is a self-orthogonal code over $R_{2^{m}}$.

Example 4.7. We give this example to obtain self-dual, self-orthogonal and quantum code parameters using some classical codes over $R_{2^{m}}$.

Case 1. $m=1, n=1$. In this case, under the conditions of Thm. 6 and Thm.7 we can take the classical code $C=\langle(0)\rangle$.

Note we can change 0 with an element in the same ideal with the norm of that element is 0 . Let $C$ be a linear code generated by the generator matrix $G=(0)$. Then we can change 0 with $\alpha, \beta$ or $\alpha+\beta+\gamma$. So, we can take the generator matrix as, for example, $G=(\alpha+\beta+\gamma)$.

- Let $G=(\alpha+\beta+\gamma)$. If we take $C_{6}=C$, in Thm.7, then we get a selforthogonal code with parameters $[4,1,4]$, and corresponding quantum code $[[4,2,2]]$.

- Let $G=(\gamma)$. If we take $C_{5}=C$ then we get a self-dual code with parameters $[4,2,2]$, and corresponding quantum code $[[4,0,2]]$. 
Case 2. $m=1, n=2$. Note we will no more take $G=(0,0)$ since it gives us a trivial self-orthogonal code.

In this case, under the conditions of Thm.6 and Thm.7 we can take the classical code $C=\langle(1,1)\rangle$.

- If we take $C_{6}=C$ then we get a self-orthogonal code with parameters $[8,1,8]$, and corresponding quantum code $[[8,6,2]]$.

- If we take $C_{5}=C$ then we get a self-orthogonal code with parameters $[8,2,4]$, and corresponding quantum code $[[8,4,2]]$.

- If we take $C_{2}=C$ then we get a self-orthogonal code with parameters $[8,3,4]$, and corresponding quantum code $[[8,2,2]]$.

- If we take $C_{1}=C$ and change one of the component 1 with $1+\alpha+\beta+\gamma$ then we get a self-dual code with parameters $[8,4,4]$, and corresponding quantum code $[[8,0,4]]$.

Thereafter, we give only special codes for some cases to understand the construction.

Example 4.8. Let $m=2, n=2$. Let $F_{4}=\left\{0,1, w, w^{2}\right\}$, where $w^{2}+w+1=$ $0, w^{3}=1$. In this case, under the conditions of Thm. 6 and Thm.7 we can take the classical a code $C$ with a generator matrix

$$
G=\left(\begin{array}{cccc}
1 & w & w^{2} & 0 \\
0 & w & 1 & w^{2}
\end{array}\right)
$$

Using this generator matrix $G$ we can easily write a generator matric for a selfdual code $C^{\prime}$ over $R_{4}$ such that

$$
C^{\prime}=\left\langle\left(\begin{array}{cccc}
1 & w(1+\alpha+\beta+\gamma) & w^{2}(1+\alpha) & w(\alpha+\beta) \\
\alpha+\beta & w(1+\alpha+\beta+\gamma) & 1+\alpha & w^{2}
\end{array}\right)\right\rangle .
$$

Here, we changed the component $w$ with $w(1+\alpha+\beta+\gamma)$, changed $w^{2}$ with $w^{2}(1+\alpha)$, changed 0 with $w(\alpha+\beta)$ since the norm of $w$ is equal to the norm of $w(1+\alpha+\beta+\gamma)$ and apply the same argument the other components.

Theorem 4.9. If $C$ is a binary self-dual code with parameters $\left[n, \frac{n}{2}, d\right]$ then there exists a binary self-dual code with parameters $[4 m n, 2 m n, \geq 2 d]$. Hence there exists a quantum code with parameters $[[4 m n, 0, \geq 2 d]]$.

Proof. Let us assume that $C$ be a binary self-dual code with generator matrix $G$. Using above method and taking $C_{1}=C$, we get a self-dual code over the $\operatorname{ring} R_{2^{m}}$ with parameters $[4 m n, 2 m n, \geq 2 d]$. Using the function $\psi$ and $\phi$, the intended is obtained.

Example 4.10. Let $C$ be the well known binary code $[2,1,2]$ with the generator matrix $G=(1,1)$. If we take the generator matrix $G^{\prime}=(1,1+\alpha+\beta+\gamma)$ for a code $C^{\prime}$, then we get a quantum code with parameters $[[8,0,4]]$.

Example 4.11. Let $C$ be the binary self-dual code with parameters $[6,3,2]$. The generator matrix $G$ of $C$ is

$$
G=\left(\begin{array}{llllll}
1 & 0 & 0 & 1 & 0 & 0 \\
0 & 1 & 0 & 0 & 1 & 0 \\
0 & 0 & 1 & 0 & 0 & 1
\end{array}\right)
$$


It is clear that the minimum weight of this code is 2 . Using the generator matrix $G$, we now construct a self-dual code. Take $k_{1}=k=3$ and $C_{1}=C$ in Thm.6. The first row of $G$ is $(1,0,0,1,0,0)$. So, we can use two elements from the set $R_{2}-M$ and can use four elements from the set $\langle\alpha\rangle \oplus\langle\beta\rangle$ since there are two reversible elements in the first row of $G$. The same manner holds for the other rows. Hence we get a generator matrix of a code $C^{\prime}$ over $R_{2}$ such as

$$
G^{\prime}=\left(\begin{array}{cccccc}
1 & 0 & 0 & 1+\alpha+\beta+\gamma & \beta & \alpha+\beta \\
0 & 1 & 0 & \beta & 1+\beta+\gamma & \beta+\gamma \\
0 & 0 & 1 & \alpha+\beta & \beta+\gamma & 1+\alpha+\gamma
\end{array}\right) .
$$

This code with parameters $[6,3,8]$ is a self-dual code over $R_{2}$. The image under the function $\psi$, that is $\psi\left(C^{\prime}\right)$, is also a binary self-dual code with parameters $[24,12,8]$. This binary self-dual code $[24,12,8]$ is an extremal self-dual code since $8=4\left\lfloor\frac{24}{24}\right\rfloor+$ 4.

Notice that we obtain a binary self-dual code whose minimum distance is 8 via a binary self-dual code whose minimum distance is 2 .

How can we determine the generator matrix $G^{\prime}$ of the code $C^{\prime}$ over $\mathbb{F}_{2^{m}}$ using the generator matrix $G$ of the code $C$ over $R_{2^{m}}$ ? Note here that $C^{\prime}=\psi(C)$.

Let

$$
G=\left(\begin{array}{c}
u_{1} \\
u_{2} \\
\vdots \\
u_{k}
\end{array}\right)_{k \times n}, \begin{array}{cccc}
u_{1}=\left(\begin{array}{cccc}
u_{11}, & u_{12}, & \ldots, & u_{1 n}
\end{array}\right) \\
u_{2}=\left(\begin{array}{cccc}
u_{21}, & u_{22}, & \ldots, & u_{2 n}
\end{array}\right) \\
\vdots \\
u_{k}=\left(\begin{array}{llll}
u_{k 1}, & u_{k 2}, & \ldots, & u_{k n}
\end{array}\right)
\end{array}
$$

where $u_{i j} \in R_{2^{m}}$. To obtain the generator matrix $G^{\prime}$, we first write the following matrix $A$;

$$
A=\left(\begin{array}{c}
u_{1} \\
(1+\alpha) u_{1} \\
(1+\beta) u_{1} \\
(1+\gamma) u_{1} \\
u_{2} \\
(1+\alpha) u_{2} \\
\vdots \\
u_{k} \\
(1+\alpha) u_{k} \\
(1+\beta) u_{k} \\
(1+\gamma) u_{k}
\end{array}\right)_{4 k \times n}
$$

Then the generator matrix $G^{\prime}$ is

$$
G^{\prime}=\left(\begin{array}{c}
\psi\left(u_{1}\right) \\
\psi\left((1+\alpha) u_{1}\right) \\
\vdots \\
\psi\left((1+\gamma) u_{k}\right)
\end{array}\right)_{4 k \times 4 n},
$$

where $\psi\left(u_{1}\right)=\left(\psi\left(u_{11}\right), \psi\left(u_{12}\right), \ldots, \psi\left(u_{1 k}\right)\right)$ and so on.

Let us assume that the matrix $G^{\prime \prime}$ is the standard form matrix of $G^{\prime}$ such that 


$$
G^{\prime \prime}=\psi(A)=\left(\begin{array}{c}
\left(\begin{array}{c}
\prime \prime \\
u_{1}^{\prime} \\
u_{2}^{\prime}
\end{array}\right) \\
\vdots \\
\left(u_{4 k}^{\prime}\right)
\end{array}\right)_{4 k \times 4 n}
$$

Let $1, w, \ldots, w^{m-1}$ be the elements of the finite field $\mathbb{F}_{2^{m}}$. We can define a matrix $B$ as

$$
B=\left(\begin{array}{c}
u_{1}^{\prime} \\
w\left(u_{1}^{\prime}\right) \\
w^{2}\left(u_{1}^{\prime}\right) \\
\vdots \\
w^{m-1}\left(u_{1}^{\prime}\right) \\
\left(u_{2}^{\prime}\right) \\
w\left(u_{2}^{\prime}\right) \\
\vdots \\
w^{m-1}\left(u_{4 k}^{\prime}\right)
\end{array}\right)_{4 k m \times 4 n}
$$

Hence, $\phi(B)$ gives us the generator matrix of a linear binary code. The dimension of the matrix $\phi(B)$ is $4 \mathrm{~km} \times 4 \mathrm{~nm}$.

Example 4.12. Let $m=2, n=2$. Let $F_{4}=\left\{0,1, w, w^{2}\right\}$, where $w^{2}+w+1=$ $0, w^{3}=1$. Let the generator matrix $G$ of a code $C^{\prime}$ over $R_{4}$ be

$$
\begin{aligned}
G & =\left(\begin{array}{cccc}
1 & \alpha+\lambda & 1+\alpha+\beta & w(\alpha+\beta) \\
\alpha+\lambda & 1+\alpha+\beta+\gamma & w(\alpha+\beta) & 1+\alpha+\beta
\end{array}\right) \\
& =\left(\begin{array}{cccc}
1 & i+k & 1+i+j & w(i+j) \\
i+k & i+j+k & w(i+j) & 1+i+j
\end{array}\right) .
\end{aligned}
$$

Then the matrix $A$ is

$$
A=\left(\begin{array}{cccc}
1 & i+k & 1+i+j & w(i+j) \\
i & 1+j & 1+i+k & w(1+k) \\
j & i+k & 1+j+k & w(1+k) \\
k & 1+j & i+j+k & w(i+j) \\
i+k & i+j+k & w(i+j) & 1+i+j \\
1+j & 1+j+k & w(1+k) & 1+i+k \\
i+k & 1+i+k & w(1+k) & 1+j+k \\
1+j & 1+i+j & w(i+j) & i+j+k
\end{array}\right)
$$


Hence the generator matrix $G^{\prime}$ is

$$
G^{\prime}=\psi(A)=\left(\begin{array}{cccccccccccccccc}
0 & 0 & 0 & 1 & 1 & 0 & 1 & 0 & 1 & 1 & 0 & 1 & w & w & 0 & 0 \\
1 & 0 & 0 & 0 & 0 & 1 & 0 & 1 & 1 & 0 & 1 & 1 & 0 & 0 & w & w \\
0 & 1 & 0 & 0 & 1 & 0 & 1 & 0 & 0 & 1 & 1 & 1 & 0 & 0 & w & w \\
0 & 0 & 1 & 0 & 0 & 1 & 0 & 1 & 1 & 1 & 1 & 0 & w & w & 0 & 0 \\
1 & 0 & 1 & 0 & 1 & 1 & 1 & 0 & w & w & 0 & 0 & 1 & 1 & 0 & 1 \\
0 & 1 & 0 & 1 & 0 & 1 & 1 & 1 & 0 & 0 & w & w & 1 & 0 & 1 & 1 \\
1 & 0 & 1 & 0 & 1 & 0 & 1 & 1 & 0 & 0 & w & w & 0 & 1 & 1 & 1 \\
0 & 1 & 0 & 1 & 1 & 1 & 0 & 1 & w & w & 0 & 0 & 1 & 1 & 1 & 0
\end{array}\right)
$$

The standard form matrix $G^{\prime \prime}$ of $G^{\prime}$ is

$$
G^{\prime \prime}=\left(\begin{array}{cccccccccccccccc}
1 & 0 & 0 & 0 & 0 & 0 & 0 & 0 & w^{2} & w & w^{2} & w^{2} & 1 & 0 & w^{2} & w \\
0 & 1 & 0 & 0 & 0 & 0 & 0 & 0 & w & w^{2} & w^{2} & w^{2} & 0 & 1 & w & w^{2} \\
0 & 0 & 1 & 0 & 0 & 0 & 0 & 0 & w^{2} & w^{2} & w^{2} & w & w^{2} & w & 1 & 0 \\
0 & 0 & 0 & 1 & 0 & 0 & 0 & 0 & w^{2} & w^{2} & w & w^{2} & w & w^{2} & 0 & 1 \\
0 & 0 & 0 & 0 & 1 & 0 & 0 & 0 & 1 & 0 & w^{2} & w & w & w^{2} & w & w \\
0 & 0 & 0 & 0 & 0 & 1 & 0 & 0 & 0 & 1 & w & w^{2} & w^{2} & w & w & w \\
0 & 0 & 0 & 0 & 0 & 0 & 1 & 0 & w^{2} & w & 1 & 0 & w & w & w & w^{2} \\
0 & 0 & 0 & 0 & 0 & 0 & 0 & 1 & w & w^{2} & 0 & 1 & w & w & w^{2} & w
\end{array}\right)
$$

The code $C^{\prime \prime}$ with the generator matrix $G^{\prime \prime}$ is a $[16,8,6]$ self dual code over $G F(4)$. Consequently, we obtain the matrix $\phi(B)$ as

$$
\phi(B)=\left(\begin{array}{l}
1,0,0,0,0,0,0,0,0,0,0,0,0,0,0,0,1,1,0,1,1,1,1,1,1,0,0,0,1,1,0,1 \\
0,1,0,0,0,0,0,0,0,0,0,0,0,0,0,0,1,0,1,1,1,0,1,0,0,1,0,0,1,0,1,1 \\
0,0,1,0,0,0,0,0,0,0,0,0,0,0,0,0,0,1,1,1,1,1,1,1,0,0,1,0,0,1,1,1 \\
0,0,0,1,0,0,0,0,0,0,0,0,0,0,0,0,1,1,1,0,1,0,1,0,0,0,0,1,1,1,1,0 \\
0,0,0,0,1,0,0,0,0,0,0,0,0,0,0,0,1,1,1,1,1,1,0,1,1,1,0,1,1,0,0,0 \\
0,0,0,0,0,1,0,0,0,0,0,0,0,0,0,0,1,0,1,0,1,0,1,1,1,0,1,1,0,1,0,0 \\
0,0,0,0,0,0,1,0,0,0,0,0,0,0,0,0,1,1,1,1,0,1,1,1,0,1,1,1,0,0,1,0 \\
0,0,0,0,0,0,0,1,0,0,0,0,0,0,0,0,1,0,1,0,1,1,1,0,1,1,1,0,0,0,0,1 \\
0,0,0,0,0,0,0,0,1,0,0,0,0,0,0,0,1,0,0,0,1,1,0,1,0,1,1,1,0,1,0,1 \\
0,0,0,0,0,0,0,0,0,1,0,0,0,0,0,0,0,1,0,0,1,0,1,1,1,1,1,0,1,1,1,1 \\
0,0,0,0,0,0,0,0,0,0,1,0,0,0,0,0,0,0,1,0,0,1,1,1,1,1,0,1,0,1,0,1 \\
0,0,0,0,0,0,0,0,0,0,0,1,0,0,0,0,0,0,0,1,1,1,1,0,1,0,1,1,1,1,1,1 \\
0,0,0,0,0,0,0,0,0,0,0,0,1,0,0,0,1,1,0,1,1,0,0,0,0,1,0,1,0,1,1,1 \\
0,0,0,0,0,0,0,0,0,0,0,0,0,1,0,0,1,0,1,1,0,1,0,0,1,1,1,1,1,1,1,0 \\
0,0,0,0,0,0,0,0,0,0,0,0,0,0,1,0,0,1,1,1,0,0,1,0,0,1,0,1,1,1,0,1 \\
0,0,0,0,0,0,0,0,0,0,0,0,0,0,0,1,1,1,1,0,0,0,0,1,1,1,1,1,1,0,1,1
\end{array}\right)
$$

Using this classical code with generator matrix $\phi(B)$, we obtain [[32,0,6]] quantum code over $\mathbb{F}_{2}$.

\section{Conclusion}

In this paper, we obtain new classes of quantum codes from classical codes over the ring $R_{2^{m}}$. To obtain these quantum codes, we define a Gray map from $R_{2^{m}}$ to $\mathbb{F}_{2^{m}}^{4}$. Also, we study on the algebraic structure of the ring $R_{2^{m}}$. We characterize cyclic, self-dual and self-orthogonal codes over this ring. 


\section{REFERENCES}

[1] Calderbank A. R., Rains, E. M., Shor, P. W., Sloane, N. J. A., "Quantum error correction via codes over $G F(4)$ ", IEEE Trans. Inform. Theory, vol. 44, pp. 1369- 1387, 1998.

[2] Kai X., Zhu S., "Quaternary construction of quantum codes from cyclic codes over $\mathbb{F}_{4}+u \mathbb{F}_{4}$ ", Int. Journal of Quantum Inf., vol. 9, no. 2, pp. 689-700, 2011.

[3] Qian J., "Quantum Codes from Cyclic Codes over $\mathbb{F}_{2}+v \mathbb{F}_{2}$ ", Journal of Information and Computational Science, vol. 10, no. 6, pp. 1715-1722, 2013.

[4] YoungJu Choie, Steven T. Dougherty, "Codes over $\Sigma_{2 m}$ and Jacobi forms over Quaternions", AAECC, vol. 15, pp. 129-147, 2004.

[5] Yildiz B., Karadeniz S., "Cyclic codes over $\mathbb{F}_{2}+u \mathbb{F}_{2}+v \mathbb{F}_{2}+u v \mathbb{F}_{2}$ ", Des. Codes Cryptogr., vol. 58, pp. 221234, 2011. (DOI: 10.1007/s10623-010-9399-3)

[6] Davidoff, G., Sarnak, P., Valette, A., Elementary number theory, group theory, and Ramanujan graphs, Cambridge University Press, 2003.

Department of Mathematics, Sakarya University, 54187, Sakarya, Türkiye

Email address: mguzeltepe@sakarya.edu.tr

Email address: meroz@sakarya.edu.tr 